\title{
Diabetes and infected foot ulcer: a survey of patients' perceptions of care during the preoperative and postoperative periods
}

Lejla Mehica, RN, MNSc, Skåne University Hospital, Department of Endocrinology, Malmö, Sweden

Magdalena Annersten Gershater, RN, $\mathrm{PhD}$, Malmö University, Faculty of Health and Society, Department of Care Science, Malmö, Sweden

Carin Alm Roijer, RN, PhD, Malmö University, Faculty of Health and Society, Department of Care Science, Malmö, Sweden

Correspondence to: Lejla Mehica, Enhetschef, Endokrinologiska kliniken, Avdelning 21, Jan Waldenströms gata 15, Plan 4, 20502 Malmö, Sweden; email: lejla.mehica@skane.se

Received: 10 May 2013

Accepted in revised form:

26 September 2013

\section{Background}

Diabetes is one of the biggest health challenges we are facing today. Amputation in patients with diabetes mellitus preceded by a foot ulcer is a serious complication. The diabetic foot can be defined as ulceration associated with neurological abnormalities, infection and various degrees of peripheral vascular disease. ${ }^{1}$

According to the International Consensus on the Diabetic Foot, healing of foot ulcers in diabetic patients is limited by multiple factors and requires a multifactorial approach. ${ }^{1}$ Furthermore, there is a need for treatment of the general condition, metabolism, infections, circulation, pressure relief, management of oedema and pain, foot surgery, topical treatment and patient education. The management of deep foot infections, hospitalisation for surgical intervention and broadspectrum antibiotic therapy are

\section{Summary}

Diabetic foot ulcer is a serious threat to the extremities and to the individual's survival. The most common risk factors for amputation are deep infection, plantar ulcer and gangrene.

The aim of this study was to measure inpatients' perception of health care quality, and to identify the health care needs of people with diabetes and infected foot ulcers during the preoperative and postoperative periods.

The study design was empirical with a quantitative approach. The short form of the Quality of care from the Patient's Perspective (Mini QPP) was used.

The results are described in four categories related to the Mini QPP model: medical technology; physical-technical conditions; identity-oriented approach; and socio-cultural atmosphere. The study participants $(n=30)$ expressed a need for continuous information, a secure and comfortable physical environment, shared decision making, and better quality of pain management.

More research about how to improve information, the environment, shared decision making, and pain control would be desirable.

Eur Diabetes Nursing 2013; 10(3): 91-95

\section{Key words}

chronic care; diabetes; foot ulcers; infection; information; nursing; pain management; preoperative care; postoperative care; surgery

essential components. Surgical interventions play a major role, and delayed surgery has been found to be associated with poor prognosis. ${ }^{1}$ These patients receive inpatient care in hospital wards. There have been no published data regarding average length of stay for this kind of surgery due to a broad variety of comorbidities in the patient group; nor are statistical data available about the number of patients who have to go through preoperative measures several times due to variations in surgical capacity.

However, at the hospital in the current study, most patients arrive in the ward two days before surgery and the majority receive surgery within one week. The ward consists of 15 beds. With a few exceptions, patients are nursed in two- or fourbed rooms with shared bathroom and shower/toilet facilities on each corridor section. The patients are cared for by registered nurses (RNs) educated to act as link nurses on the ward, and they also have access to a diabetes specialist nurse. Assistant nurses are trained in diabetic foot ulcer wound management.

\section{Nursing}

Preoperative measures. The fundamental characteristics of patientcentred care were identified as the individualisation of patient care and the involvement of the patient in their care through information and shared decision making. ${ }^{2}$ All preoperative measures take place in the ward. Everyone who will be undergoing a surgical procedure, no matter how minor, has some degree of anxiety about the procedure, its outcomes and potential complications. The outcomes of surgical procedures are almost always improved by a reasonably long preoperative planning period. This gives the surgical team and the patient maximum opportunity for physical and 
Diabetes and infected foot ulcer: patients' perceptions of preoperative and postoperative care

technical preparation. However, excessively long preoperative periods may be associated with increased amounts of worry, anxiety, and stress; these factors can have a negative impact on surgical outcomes. Patients should be given individualised information about their health, methods of examination, care and treatment. Effective information aims to meet individuals' information needs to enable them to enhance recovery, independence and well-being. ${ }^{3}$

Postoperative measures. Returning to the ward after an operation means patients' vital signs have become stable. 4 During this time, RNs play a key role in: effectively managing the patient's pulmonary, neurological and circulatory status; preventing pressure ulcers; controlling pain, nausea and/or vomiting; and patient safety. ${ }^{4}$ In addition to the wounds and tissue damage inflicted by the surgery itself, postoperative patients are at risk of a number of complications caused by their decreased mobility. Early complications may include partial lung collapse that results from shallow, painful breathing, ${ }^{5}$ bladder infections from indwelling catheters, local inflammation of the healing wound, ${ }^{6}$ and inflammation caused by blood clots developing in nonmoving lower extremities. ${ }^{7}$ Pressure ulcers, or bedsores, develop at pressure points in patients who are unable or unwilling, because of pain, to shift their positions in the bed. ${ }^{4}$ Necrosis, cell death, can occur very rapidly in these ulcers, resulting in the development of potentially large masses of dead and dying tissue, which provide a breeding ground for bacteria. For these reasons, bedsores can be life threatening. Their prevention is one of the main priorities of the nurses in the postoperative period. Poor nutritional status is a major risk factor for their development, ${ }^{8}$ and many nutritional interventions are known to be helpful. ${ }^{9-11}$

Brener ${ }^{4}$ described the complexity of the RNs' role in postoperative care: to assist, coordinate and observe the results of the medical treatment; to observe the patient's clinical condition in terms of improvement and deterioration; to support the patient's capacity for recovery and healing; and teaching patients to live with their disease and its possible consequences.

The nursing process can be viewed as a problem-solving and decision-making process performed by RNs who have formulated individual care plans including nursing diagnosis, goals and interventions. RNs' responsibility is also about coordinating the team, ${ }^{12}$ the use of off-loading equipment, and insertion and care of peripheral venous catheters. Furthermore, RNs are responsible for administration of intravenous antibiotic medication, pain management, nutrition, and maintaining blood glucose control prior to discharge. Meeting the need for individually adjusted education and improved communication methods is also dependent on the RNs. ${ }^{13}$

\section{The quality of care}

Health care should not be accessed exclusively by clinical and economic efficiency, without taking into account social, ethical and humanitarian aspects. International and governmental developments affecting health care, and the increasing patient influence should also be taken into account. ${ }^{14}$ The fundamental principle of nursing is to meet the patient's desires and needs. Patients' perceptions of what is important in health care should be seen as an aspect of quality of care. ${ }^{15}$ Organisational strategies with the goal of improving the safety of patients undergoing surgical procedures are important parts of the process. Better planned ward rounds, better communication and teamwork between clinical disciplines, using tools like checklists or singlebed rooms, would improve patients' perception of quality of care.

\section{Aims}

The purpose of this study was to identify patients' perception of health care quality and to identify the health care needs of people with diabetes and infected foot ulcers during the preoperative and postoperative phases in an inpatient hospital ward. The focus was on two main issues: medical-technical skills and physical-technical conditions.

\section{Methods}

\section{Design and setting}

The study was designed as a baseline measurement, an empirical study with quantitative data. The questionnaire used contained 49 questions, including two open questions that give patients the opportunity to write down their own experiences. The study was carried out at a department of endocrinology in a university hospital in southern Sweden during 2011. As stated above, the ward is a 15bed unit; there are two senior physicians, three junior doctors, five RNs, four health care assistants, one physiotherapist, one nutritionist and one social worker caring for the patients.

\section{Study population and criteria}

Study inclusion criteria were:

- Patients $>18$ years of age.

- Patients with a diagnosis of diabetes. - Patients undergoing amputation of the toe or forefoot in an operating room due to acute diabetic foot infection.

Patients were excluded if they were:

- Hospitalised for conservative treatment with antibiotics only.

- Undergoing reconstructive vascular surgery or percutaneous transluminal angioplasty only. 


\begin{tabular}{l|l} 
Characteristics & \\
\hline Men; no. (\%) & $22(73)$ \\
\hline Women; no. (\%) & $8(27)$ \\
\hline Age; mean years \pm SD & $65 \pm 12.9$ \\
\hline $\begin{array}{l}\text { Insulin treatment; no. (\%) } \\
\text { Duration of diabetes; } \\
\text { mean years } \pm \text { SD }\end{array}$ & $14 \pm 22.9$ \\
\hline Born in Sweden; no. (\%) & $23(77)$ \\
\hline $\begin{array}{l}\text { Self-reported employment } \\
\text { status; no. (\%) }\end{array}$ & $25(83)$ \\
\hline
\end{tabular}

Table 1. General patient characteristics at enrolment, $n=30$

- Undergoing major amputation above the ankle.

- Had a diagnosis of dementia.

\section{Instrument}

The questionnaire Mini QPP (short form of the Quality of care from the Patient's Perspective) was used in an Improve.IT computer program. Developed by Wilde Larsson and Larsson ${ }^{15}$ in order to measure quality of care, it consists of a 49-item questionnaire including three different response scales. Computerbased, the Mini QPP makes two conclusions on every single issue: perceived reality and subjective meaning. Both assessments are done on a 4-point Likert scale. The questionnaire has been tested for validity and reliability. ${ }^{16}$

Questions relating to home care were considered as irrelevant based on the aim of the study and so were omitted. A total of eight questions from the original version considering home care after discharge from hospital were removed. Five specific issues regarding demographics were added. In total, 46 questions were used. Validity and reliability were not influenced, according to Wilde Larsson and Larsson. ${ }^{15}$ No gatekeeper was used.

\section{Data collection}

Patients (n=35) who were hospitalised in the inpatients' ward $(\mathrm{n}=15$ beds) who met inclusion/exclusion criteria were consecutively asked to participate in the study by a diabetes specialist nurse. Two patients with a diagnosis of dementia were subsequently excluded. The interviews took place on the day of discharge. Those who could not read and understand Swedish communicated through qualified interpreters. Both oral and written information (irrespective of language) was provided.

Thirty-three patients were asked to participate. All the invited patients agreed to participate and signed the informed consent form. The questionnaire forms were answered at the hospital in a separate room with only the patient, the study nurse and the qualified interpreter, if needed, present. In three cases, the questionnaires were answered through the qualified interpreters. Contact was established with a health care certified translation office in Malmö, Sweden, and three certified interpreters in Bosnian, Arabic and Persian were used.

\section{Data analysis}

Data from the Mini QPP were processed using the Windows-based computer program, Google docs. The answers are stored securely in a database on the internet and are available through a web-reader protected by password. No names or other identifying information were recorded.

Quality of care is composed of four interdependent dimensions, which are an inseparable part of an entirety in the QPP. The answers were analysed by the QPP model's four categories:

- Medical-technical competence (correct examination, correct diagnosis, correct treatment and adequate care).

- Physical-technical conditions (necessary medical equipment; adequate nutrition; safety, secure and comfortable physical environment).

- Identity-oriented approach (compassion and commitment, mutual respect and understanding, honesty, climate of cooperation, evident responsibility).

- Socio-cultural atmosphere (social interaction, possibility to influence practices at the ward, privacy, good atmosphere in general).

The two open questions were analysed based on manifest content analysis according to Krippendorff. ${ }^{16}$ As the patients answered using very short sentences, the results are presented as group statements.

\section{Ethical considerations}

Malmö University Advisory Committee for Research Ethics in Health Education approved the study in January 2011 (Dnr HS60- 10/987:19).

\section{Results}

\section{Demographic data}

General patient characteristics at enrolment are shown in Table 1. Study participants numbered 30 in all (22 men and 8 women).

\section{Medical-technical skills}

Thirty percent $(n=9)$ received their surgery within a week (Table 2). The majority of patients $63 \%(n=19)$ had to wait for surgery between one week and one month, and $7 \%(\mathrm{n}=2)$ were waiting for more than one month. Fifty percent of the patients $(n=15)$ described the waiting period as 'neither easy nor difficult' and 17\% $(n=5)$ as 'very difficult'. This issue had a great subjective importance for $77 \%$ of the respondents $(n=23)$.

Fifty-three percent $(n=16)$ of the respondents felt great anxiety or fear about the surgical procedure. Forty percent $(n=12)$ identified their physical condition as relatively poor and $13 \%(n=4)$ as very poor.

Twenty-seven percent $(n=8)$ were dissatisfied with the level of pain 
Diabetes and infected foot ulcer: patients' perceptions of preoperative and postoperative care

relief received. Effective pain relief was reported as the most important matter for $83 \% \quad(n=25)$ of the respondents.

\section{Physical-technical conditions}

See Table 2. Seventy percent $(n=21)$ of the respondents lacked information about nutrition in conjunction with surgery. With regard to a comfortable physical environment, $30 \%$ $(n=9)$ reported that they did not have a comfortable bed. Seventyseven percent $(n=23)$ lacked an opportunity to speak in private with both physicians and nurses. Having the opportunity to speak with health care professionals in general had great subjective importance: $80 \%(\mathrm{n}=24)$ for physicians in private and $77 \%(\mathrm{n}=23)$ for nurses in private.

\section{Identity-oriented approach}

Thirty percent $(n=9)$ of the respondents considered that physicians did not understand how they perceived their situation - a very important issue for $80 \%(n=24)$. On the other hand, $70 \% \quad(n=21)$ of the respondents experienced that physicians responded to their needs with respect and commitment.

Seventy-seven percent $(n=23)$ of the respondents lacked information about whom they should contact if they had questions about the foot surgery.

\section{Socio-cultural atmosphere}

Treatment and care should take into account patients' needs and preferences in formulating a plan of care that respects and values the patient's uniqueness: $13 \% \quad(n=4)$ of the respondents strongly agreed, $17 \%$ $(\mathrm{n}=5)$ almost agreed and 27\% ( $\mathrm{n}=8)$ partly agreed.

\section{Results of the open-ended questions}

Ninety percent $(n=27)$ of the participants responded to the two openended questions.

\begin{tabular}{l|l} 
Characteristics & No. (\%) \\
\hline \multicolumn{2}{l|}{ Medical-technical skills } \\
\hline Satisfied with pain relief & $22(73)$ \\
\hline Waiting time <1 week & $9(30)$ \\
\hline Physical-technical conditions \\
\hline $\begin{array}{l}\text { Satisfied with information } \\
\text { about nutrition }\end{array}$ & $9(30)$ \\
\hline $\begin{array}{l}\text { Comfortable physical } \\
\text { environment }\end{array}$ & $21(70)$ \\
\hline \multicolumn{2}{l}{ Socio-cultural atmosphere } \\
\hline Pleasant atmosphere & $23(77)$ \\
on the ward &
\end{tabular}

Table 2. Overview of participants' responses relating to medical-technical skills, physical-technical conditions, and socio-cultural atmosphere

Medical-technical skills. Respondents were satisfied with the health care professionals' competence (specialist doctors, senior physicians, junior doctors, RNs, health care assistants, physiotherapist, nutritionist and social worker) in diabetes and wound healing. Improvement in pain management $(13 \%, \mathrm{n}=4)$, surgical waiting times $(7 \%, \mathrm{n}=2)$, and continuous information given by the same physician/nurse $(33 \%, \mathrm{n}=10)$ were some of the suggestions provided by participating patients.

Physical-technical conditions. There were suggestions for improvement of the ward environment, hospital overcrowding and lack of appropriate hygiene from $43 \%(n=13)$ of the respondents.

Identity-oriented approach. Treatment satisfaction was reported by $47 \% \quad(n=14)$ of the respondents. There were 10 answers describing the staff as friendly and helpful. Twentyseven percent $(n=8)$ had suggestions for improved intercommunication and information flow tailored to the patients' needs such as 'information responsible officer' or better translation service.

Socio-cultural atmosphere. Seventyseven percent $(n=23)$ were satisfied with the ward atmosphere. According to $13 \%(\mathrm{n}=4)$ of the respondents, patients should have the opportunity to be involved in decisions about treatment and care, and more than half $(53 \% ; \mathrm{n}=16)$ reported this as a very important matter.

\section{Discussion}

The findings show that information, pain control, physical environment and shared decision making in relation to surgery in infected diabetes foot ulcers need to be improved. There are several factors that affect this: a combination of medical-technical skills, physical-technical conditions, an identity-oriented approach and socio-cultural atmosphere. The development of local guidelines ensuring the appropriate use of evidence-based methods and improved teamwork on the ward seems to be the most important factor in structured health care management. This has, to our knowledge, not been shown in previous studies regarding patients' perceptions of care in connection with amputation for infected diabetes foot ulcers. However, other patient groups have been studied regarding pre- and postoperative care, with similar conclusions. ${ }^{4}$

The present study identified that patients in this cohort do not receive sufficient pain relief. Preoperative anxiety and symptoms of depression may cause reactions that result in an increase in intraoperative consumption of anaesthetics and greater postoperative demand for analgesics, and seem to have a profound influence on the immune system and on the development of infections. To prevent preoperative anxiety there is a need to find out the incidence of 
anxiety in preoperative patients and to identify its association with good communication. ${ }^{17}$ This study was an attempt to explore this relationship.

Several studies have found that the introduction of guidelines for acute pain management using a nurse-based anaesthesiologist-supervised model resulted in more adequate pain management routines, and better patient satisfaction. Additionally, information about postoperative pain control and confidence in pain management among RNs on the wards increased. ${ }^{1,18}$ This issue should be the main priority in the quality work of every similar ward caring for patients with diabetes and infected foot ulcers. Together with improved patient information, these constitute the issues we have to face with solution-oriented nursing: that is, working on improving staff competence by education of RNs and nurse assistants; introducing specified guidelines regarding pre- and postoperative care; optimising the physical environment; and promoting patient participation in decision making and care.

\section{Methodological considerations}

Reliability. Each item was evaluated in two ways by the respondent: to measure perceived reality of quality of care and subjective importance. ${ }^{16}$ The instrument has been used in a number of previous studies measuring quality of care. ${ }^{9-11}$

Validity. The Mini QPP was used in order to identify patients' perception of quality of care. The fact that an external study nurse was not involved in direct patient care, and that the questionnaires were distributed to patients after treatment had finished, probably contributes to reducing bias in patients' responses which might have resulted from any sense of obligation towards the ward nurses.

Many patients stated that it was sometimes difficult to understand the multiple-choice answers, especially since those relating to subjectivity were quite similar. The patients who could not read and understand Swedish communicated through qualified interpreters to enable correct understanding.

Two of the 30 respondents asked for help using the computer and it is difficult to state whether this affected the results or not. According to the Mini QPP authors, ${ }^{15}$ it is important that the interviewer and not the family help the patient to understand the issues, because family members may have an opinion on health care which is different from that of the patient. Therefore, in the present study, no family members were invited. In addition, high response rate and 'honest' answers depend on whether patients feel confident that their anonymity is protected. Based on the results of the baseline study, several care improvement interventions are planned: ward guidelines and standards focusing on patient information and pain management; staff education; and a follow-up study to evaluate the effects of the interventions.

\section{Conclusions}

People with diabetic foot problems should have the opportunity to make informed decisions about their care and treatment, in partnership with their health care professionals. Surgery was associated with risks and concerns, long recovery and rehabilitation, limited mobility and changes in lifestyle and family situations. Good communication between health care professionals and patients is essential.

Quality in nursing can be improved by:

- Effective continual information.

- Better pain management.

- A secure and comfortable physical environment.

- Better shared decision making for the patients.

\section{Declaration of interests}

There are no conflicts of interest declared.

\section{References}

1. International Consensus on the Diabetic Foot and Practical Guidelines on the Management and the Prevention of the Diabetic Foot. Amsterdam: International Working Group on the Diabetic Foot, 2011.

2. Pelzang R. Time to learn: understanding patient-centred care. Br J Nurs 2010;19(14): $12-7$.

3. Labott S, et al. Health psychology clinical experiences. Department of Psychiatry, University of Illinois at Chicago, 2010.

4. Brener ZR. Preventing postoperative complications. Nurse Manag 2000;31(12):17-22.

5. Westerdahl E, et al. Deep-breathing exercises reduces atelectasis and improve pulmonary function after coronary artery bypass surgery. Chest 2005;128(5):3482-8.

6. Larsen JW, et al. Guidelines for the diagnosis, treatment and prevention of postoperative infections. Infect Dis Obstet Gynecol 2003; 11(1):65-70.

7. Vucic N, et al. Therapy and prevention of deep venous thrombosis and pulmonary embolism in gynaecology and obstetrics. Acta Med Croatica 2003;57(2):123-30.

8. Domini LM, et al. Nutritional status and evolution of pressure sores in geriatric patients. J Nutr Health Aging 2005;9(6):446-54.

9. Desneves KJ, et al. Treatment with supplementary arginine, vitamin $\mathrm{C}$ and zinc in patients with pressure ulcers: A randomised controlled trial. Clin Nutr 2005;24(6): 979-87.

10. Patel GK. The role of nutrition in the management of lower extremity wounds. Int $J$ Low Extrem Wounds 2005;4(1):12-22.

11. Braga M, Gianotti L. Preoperative immunonutrition: Cost-benefit analysis. JPEN J Parenter Enteral Nutr 2005;29(1 Suppl):S57-S61.

12. Jansson I, et al. Obtaining a foundation for nursing care at the time of patient admission: a grounded theory study. Open Nurs 2009;3:56-64

13. Gershater M, et al. Complexity of factors related to outcome of neuropathic and neuroischaemic/ischaemic diabetic foot ulcers: a cohort study. Diabetologia 2009; 52(3):398-407.

14. Williams SJ, Calnan M. Key determinants of consumer satisfaction with general practice. Fam Pract 1991;8(3):237-42.

15. Wilde Larsson B, Larsson G. Quality of care from the Patient's Perspective Questionnaire (QPP). In Commissioned Reviews of 250 Psychological Tests. Maltby J, et al. (eds). Lampeter, Wales: Edwin Mellen Press, 2000; 113-6.

16. Krippendorff K. Content analysis: an introduction to its methodology. Thousand Oaks, CA: Sage, 2004.

17. Caumo W, et al. Risk factors for postoperative anxiety in adults. Anaesthesia 2001;56(8): 720-8.

18. De Rond $\mathrm{M}$, et al. The implementation of a pain monitoring programme for nurses in daily clinical practice: results of a follow-up study in five hospitals. I Adv Nurs 2001; 35(4):590-8. 